\title{
ESTIMATES FOR THE FIRST EIGENFUNCTION OF LINEAR EIGENVALUE PROBLEMS VIA STEINER SYMMETRIZATION
}

\author{
Francesco Chiacchio
}

\begin{abstract}
By means of Steiner symmetrization we get some estimates for the first eigenfunction of a class of linear problems, having as prototype the Laplacian with Dirichlet boundary conditions.
\end{abstract}

\section{Introduction}

The model problem we consider here is a very classical one: the fixed membrane problem, i.e.

$$
\begin{cases}-\Delta u=\lambda u & \text { in } \Omega \\ u=0 & \text { on } \partial \Omega\end{cases}
$$

where $\Omega$ is an open, bounded and connected subset of $\mathbb{R}^{n}$.

As well-known, the symmetrization methods have turned out to be a remarkable tool for the study of elliptic and parabolic equations. Many monographs, indeed, deal with this subject, see for instance $[\mathbf{2 1}],[\mathbf{6}]$, $[\mathbf{1 9}],[\mathbf{1 7}]$ and $[\mathbf{1 8}]$. For exhaustive references on this topic, we refer the reader to the detailed bibliographies contained in $[\mathbf{1 8}]$ and in $[\mathbf{2 3}]$.

In particular, Schwarz symmetrization has allowed to obtain various estimates for the first eigenfunction $u$ and for the eigenvalues $\lambda_{i}$ of (1.1), see, for instance, [16]. Let us briefly describe some results in this direction, due to Chiti, which are close to ours. Let $u^{\star}$ denotes the Schwarz rearrangement of $u$ and let $S_{\lambda_{1}}$ be the ball of $\mathbb{R}^{n}$ centered at the origin, such that the Laplacian with Dirichlet boundary conditions has its first eigenvalue equal to $\lambda_{1}$ and finally $z$ a corresponding eigenfunction. In [11], Chiti proved that, if $u$ and $z$ are normalized in a suitable way,

2000 Mathematics Subject Classification. 35J25, 35B05, 35P05.

Key words. Steiner symmetrization, comparison results, linear eigenvalue problems. 
then $u^{\star}$ can be pointwise estimated in terms of $z$. This result relies on the Talenti's Theorem (see $[\mathbf{2 2}]$ ) which ensures that

$$
-u^{* \prime}(s) \leq \lambda_{1} n^{-2} \omega_{n}^{-2 / n} s^{-2+2 / n} \int_{0}^{s} u^{*}(\sigma) d \sigma,
$$

where $\omega_{n}$ is the measure of the unit ball of $\mathbb{R}^{n}$ and $u^{*}$ is the decreasing rearrangement of $u$

$$
u^{*}(s)=\sup \{t \geq 0: \mu(t)>s\}
$$

where, finally, $\mu$ is the distribution function of $|u|$.

Chiti's results have been generalized to nonlinear equations (see, for instance, $[\mathbf{1}]$ and $[\mathbf{8}]$ ) and to the eigenvalue problem for the Hermite equation via the Gaussian symmetrization (see $[\mathbf{7}]$ ). We finally recall that Chiti's type estimates were also used by Ashbaugh and Benguria in order to solve the well-known Payne-Pólya-Weinberger conjecture (see [3]) and its generalization on the $n$-dimensional sphere $\mathbb{S}^{n}$ (see [4]).

Now, let us consider problem (1.1) for domains $\Omega \subset \mathbb{R}_{x}^{n} \times \mathbb{R}_{y}^{m}$ having $n$-dimensional cross sections of constant thickness. Or, more precisely, assume that for any fixed $y$ in $\Omega^{\prime \prime}$, where $\Omega^{\prime \prime}=\left\{y \in \mathbb{R}^{m}: \exists x \in \mathbb{R}^{n}\right.$ : $(x, y) \in \Omega\}$, it holds ${ }^{1}$

$$
\left|\left\{x \in \mathbb{R}^{n}:(x, y) \in \Omega\right\}\right|_{n}=L \in(0,+\infty) .
$$

In this case instead of Schwarz symmetrization, which should transform $\Omega$ into $\Omega^{\star}$, the ball of $\mathbb{R}^{n+m}$ centered at the origin having the same measure as $\Omega$, it is more natural to use Steiner symmetrization with respect to the variables $x_{i}$. In this way the symmetrized set turns out to be the cylinder $B_{R} \times \Omega^{\prime \prime}$, where $B_{R}$ is the ball of $\mathbb{R}^{n}$, centered at the origin whose measure is $L$. Being this set closer to the original domain with respect to $\Omega^{\star}$, with this procedure one can obtain sharper estimates for $u$. This last point is the aim of this paper.

Hence, let $\Omega$ be an open, bounded and connected subset of $\mathbb{R}_{x}^{n} \times \mathbb{R}_{y}^{m}$ verifying (1.3) and let $\lambda_{1}$ be the first eigenvalue of (1.1) and $u=u(x, y)$ be a nonnegative corresponding eigenfunction. For $y$ in $\Omega^{\prime \prime}$, we consider the function $u(\cdot, y)$, whose decreasing rearrangement will be denoted with $u^{*}(s, y)$.

\footnotetext{
${ }^{1}$ Here and throughout $|\cdot|_{n}$ will stand for the $n$-dimensional Lebesgue measure.
} 
Our starting point is, in place of (1.2), the following differential inequality proved in $[\mathbf{2}]$ (see also $[\mathbf{5}]$ and $[\mathbf{1 3}]$ )

$$
\begin{cases}-U_{s s}-n^{-2} \omega_{n}^{-2 / n} s^{-2+2 / n} \Delta_{y} U & \\ \leq \lambda_{1} n^{-2} \omega_{n}^{-2 / n} s^{-2+2 / n} U & \forall(s, y) \in(0, L) \times \Omega^{\prime \prime} \\ U(0, y)=U_{s}(L, y)=0 & \forall y \in \Omega^{\prime \prime} \\ U(s, y)=0 & \forall(s, y) \in(0, L) \times \partial \Omega^{\prime \prime},\end{cases}
$$

where $U(s, y)=\int_{0}^{s} u^{*}(\sigma, y) d \sigma$.

Now let $B(0, r)$ the ball of $\mathbb{R}^{n}$ such that $\lambda_{1}$ is also the first eigenvalue of

$$
\begin{cases}-\Delta v=\lambda v & \text { in } B(0, r) \times \Omega^{\prime \prime} \\ v=0 & \text { on } \partial\left(B(0, r) \times \Omega^{\prime \prime}\right),\end{cases}
$$

and let $v$ be a corresponding positive eigenfunction.

As we will see

$$
|B(0, r)|=l \leq L,
$$

and moreover the following equality holds

$$
\begin{cases}-V_{s s}-n^{-2} \omega_{n}^{-2 / n} s^{-2+2 / n} \Delta_{y} V & \\ =\lambda_{1} n^{-2} \omega_{n}^{-2 / n} s^{-2+2 / n} V & \forall(s, y) \in(0, l) \times \Omega^{\prime \prime} \\ V(0, y)=V_{s}(l, y)=0 & \forall y \in \Omega^{\prime \prime} \\ V(s, y)=0 & \forall(s, y) \in(0, l) \times \partial \Omega^{\prime \prime}\end{cases}
$$

where $V(s, y)=\int_{0}^{s} v^{*}(\sigma, y) d \sigma$.

We prove that it is possible to normalize $u$ and $v$ in such a way to have

$$
\int_{0}^{l} u^{*}(\sigma, y) d \sigma \leq \int_{0}^{l} v^{*}(\sigma, y) d \sigma, \quad \forall y \in \Omega^{\prime \prime},
$$

moreover, once (1.7) is fulfilled, then

$$
\int_{0}^{s} u^{*}(\sigma, y) d \sigma \leq \int_{0}^{s} v^{*}(\sigma, y) d \sigma, \quad \forall(s, y) \in(0, l) \times \Omega^{\prime \prime}
$$

Note that condition (1.7) is not trivially verified, since

$$
\lim _{y \rightarrow \partial \Omega^{\prime \prime}} \int_{0}^{l} u^{*}(\sigma, y) d \sigma=\lim _{y \rightarrow \partial \Omega^{\prime \prime}} \int_{0}^{l} v^{*}(\sigma, y) d \sigma=0 .
$$


The result above is then extended to the following class of elliptic problems

$$
\left\{\begin{array}{l}
-\left(a_{i j}(x, y) u_{x_{i}}\right)_{x_{j}}-\left(b_{h k}(y) u_{y_{h}}\right)_{y_{k}}=\lambda u \text { in } \Omega \\
u \in H_{0}^{1}(\Omega)
\end{array}\right.
$$

where

i) $a_{i j}(x, y)=a_{j i}(x, y)$ and $b_{h k}(y)=b_{k h}(y)$, a.e. $(x, y) \in \Omega$,

ii) $|\xi|^{2}+\nu|\eta|^{2} \leq a_{i j}(x, y) \xi_{i} \xi_{j}+b_{h k}(y) \eta_{h} \eta_{k} \leq \Theta\left(|\xi|^{2}+\nu|\eta|^{2}\right)$, for some $\nu>0$ and $\Theta>1, \forall(\xi, \eta) \in \mathbb{R}^{n} \times \mathbb{R}^{m}$ and a.e. $(x, y) \in \Omega$,

iii) the coefficients $b_{h k}(y)$ are analytic in $\overline{\Omega^{\prime \prime}}$.

The paper is organized as follows. In Section 2 we recall some definitions and properties about Steiner rearrangement. We also mention the one-dimensional Hardy inequality, since it will turn out to be a useful tool in proving that the operator appearing at the left hand side of the equation in (1.6) is compact in a weighted Sobolev space. In Section 3 we provide the comparison Theorem for the Laplacian. In that case, by simple factorization arguments, we show that the function $V$ can be written explicitly in terms of Bessel functions and the first eigenfunction of the following problem

$$
\begin{cases}-\Delta_{y} w=\mu w & \text { in } \Omega^{\prime \prime} \\ w=0 & \text { on } \partial \Omega^{\prime \prime}\end{cases}
$$

In the last section, the result is proved for problems of the type (1.9). In this case, via an approximation procedure, we overcome the difficulty arising from the presence of nonsmooth coefficients in the equation.

\section{Preliminaries}

Let $\Omega$ be an open, bounded and connected subset of $\mathbb{R}_{x}^{n} \times \mathbb{R}_{y}^{m}$ and let $u=u(x, y)$ be a function defined in $\Omega$. We will denote by $\Omega^{\prime \prime}$ the projection of $\Omega$ on the linear manifold $\{x=0\}$ i.e.

$$
\Omega^{\prime \prime}=\left\{y \in \mathbb{R}^{m}: \exists x \in \mathbb{R}^{n}:(x, y) \in \Omega\right\} .
$$

In the sequel $|\cdot|_{n}$ will stand for the $n$-dimensional Lebesgue measure, repeated indices mean summation, $C$ will denote a positive constant whose value may change from line to line and the following notation will 
be in force

$$
\begin{aligned}
B_{r} & =\left\{x \in \mathbb{R}^{n}:|x|<r\right\} \\
\omega_{n} & =|B(0,1)|_{n} \\
I_{l} & =(0, l) \\
g(s) & =n^{-2} \omega_{n}^{-\frac{2}{n}} s^{-2+\frac{2}{n}} \\
D_{x} u & =\left(u_{x_{1}}, \ldots, u_{x_{n}}\right) \\
D_{y} u & =\left(u_{y_{1}}, \ldots, u_{y_{m}}\right) \\
\Delta_{x} u & =u_{x_{i} x_{i}} \\
\Delta_{y} u & =u_{y_{h} y_{h}} \\
\Delta u & =\Delta_{x} u+\Delta_{y} u .
\end{aligned}
$$

For any fixed $y$ in $\Omega^{\prime \prime}, \mu(t, y)$ will denote the distribution function of $|u|(\cdot, y)$. The Steiner rearrangement of $u$ with respect to $x$ is given by

$$
u^{\sharp}(x, y)=u^{*}\left(\omega_{n}|x|^{n}, y\right) \text {, with }(x, y) \in \Omega^{\sharp},
$$

where $\Omega^{\sharp} \subset \mathbb{R}^{n} \times \mathbb{R}^{m}$ is the domain uniquely defined by the following relations

$$
\left\{x \in \mathbb{R}^{n}:(x, y) \in \Omega^{\sharp}\right\}=B_{r_{y}}, \quad \forall y \in \Omega^{\prime \prime},
$$

with $r_{y}$ such that

$$
\left|B_{r_{y}}\right|_{n}=\left|\left\{x \in \mathbb{R}^{n}:(x, y) \in \Omega\right\}\right|_{n},
$$

and finally

$$
\left\{x \in \mathbb{R}^{n}:(x, y) \in \Omega^{\sharp}\right\}=\emptyset, \quad \forall y \in \mathbb{R}^{m} \backslash \Omega^{\prime \prime} .
$$

The equimisurability of $u$ and $u^{\sharp}$ ensures that Steiner symmetrization leaves the $L^{p}$-norms of a function unaltered. On the other hand, the following Pólya-Szegö principle holds (see, for instance, [17] and [12]).

Theorem 1. For any $p \geq 1$, we have

$$
\int_{\Omega}|D u|^{p} d x d y \geq \int_{\Omega^{\sharp}}\left|D u^{\sharp}\right|^{p} d x d y, \text { for any } u \in W_{0}^{1, p}(\Omega) .
$$

We end this section by recalling the simplest version of the Hardy inequality (see $[\mathbf{2 0}]$ ).

Theorem 2. For any $u \in H^{1}\left(I_{l}\right)$, with $u(0)=0$, it holds

$$
\int_{I_{l}}\left|u^{\prime}(t)\right|^{2} d t \geq \frac{1}{4} \int_{I_{l}} \frac{u^{2}}{t^{2}} d t .
$$




\section{The case of the Laplacian}

Let $\Omega$ be an open, bounded, connected and Lipschitz subset of $\mathbb{R}^{n+m}$ such that

$$
\left|\left\{x \in \mathbb{R}^{n}:(x, y) \in \Omega\right\}\right|_{n}=L \in(0,+\infty), \quad \forall y \in \Omega^{\prime \prime},
$$

furthermore, we will assume that $\Omega^{\prime \prime}$ is a $C^{2, \alpha}$ domain, for some positive $\alpha$.

Let $\lambda_{1}$ be the first eigenvalue of the problem

$$
\begin{cases}-\Delta w=\lambda w & \text { in } \Omega \\ w=0 & \text { on } \partial \Omega,\end{cases}
$$

and $u$ a corresponding eigenfunction.

Let us fix $R>0$ such that $\lambda_{1}$ is the first eigenvalue of the symmetrized problem

$$
\begin{cases}-\Delta v=\lambda v & \text { in } B_{R} \times \Omega^{\prime \prime} \\ v=0 & \text { on } \partial\left(B_{R} \times \Omega^{\prime \prime}\right),\end{cases}
$$

and let us denote by

$$
l=\left|B_{R}\right|_{n} .
$$

A result contained in $[\mathbf{2}]$ (see also $[\mathbf{1 3}]$ ) ensures that

$$
\begin{cases}-U_{s s}-g(s) \Delta_{y} U \leq \lambda_{1} g(s) U & \text { in } I_{L} \times \Omega^{\prime \prime} \\ U(0, y)=U_{s}(L, y)=0 & \forall y \in \Omega^{\prime \prime} \\ U(s, y)=0 & \forall(s, y) \in I_{L} \times \partial \Omega^{\prime \prime}\end{cases}
$$

and

$$
\begin{cases}-V_{s s}-g(s) \Delta_{y} V=\lambda_{1} g(s) V & \text { in } I_{l} \times \Omega^{\prime \prime} \\ V(0, y)=V_{s}(l, y)=0 & \forall y \in \Omega^{\prime \prime} \\ V(s, y)=0 & \forall(s, y) \in I_{l} \times \partial \Omega^{\prime \prime}\end{cases}
$$

where respectively

$$
U(s, y)=\int_{0}^{s} u^{*}(\sigma, y) d \sigma, \quad(s, y) \in I_{L} \times \Omega^{\prime \prime},
$$

and

$$
V(s, y)=\int_{0}^{s} v^{*}(\sigma, y) d \sigma, \quad(s, y) \in I_{l} \times \Omega^{\prime \prime} .
$$


Now, let $\mu_{1}$ be the first eigenvalue of the problem

$$
\begin{cases}-\Delta_{y} w=\mu w & \text { in } \Omega^{\prime \prime} \\ w=0 & \text { on } \partial \Omega^{\prime \prime}\end{cases}
$$

and let us denote by $j_{p, k}$ the kth positive zero of the Bessel function $J_{p}$.

The next lemma gives some information about the symmetrized problem (3.3).

Lemma 1. Under the assumptions and notation introduced below, it holds that

$$
\begin{gathered}
l \leq L, \\
l=\frac{\omega_{n} j_{\frac{n}{2}}^{2}-1,1}{\left(\lambda_{1}-\mu_{1}\right)^{n / 2}},
\end{gathered}
$$

and, up to a multiplicative factor,

(3.9) $V(s, y)=\chi(y) \int_{0}^{s}\left(\frac{\sigma}{\omega_{n}}\right)^{\frac{2-n}{2 n}} J_{\frac{n}{2}-1}\left(\left(\lambda_{1}-\mu_{1}\right)^{1 / 2}\left(\frac{\sigma}{\omega_{n}}\right)^{1 / n}\right) d \sigma$

where $\chi=\chi(y)$ is an eigenfunction of (3.6) corresponding to $\mu_{1}$.

Proof: The variational characterization of the first eigenvalue, together with Pólya-Szegö principle (2.1), gives immediately (3.7).

Now, let us show (3.8) and (3.9). By separating the variables, we can assume that

$$
V(s, y)=S(s) \chi(y) .
$$

The equation in (3.3) becomes

$$
-g^{-1}(s) \frac{S^{\prime \prime}}{S}-\frac{\Delta_{y} \chi}{\chi}=\lambda_{1} .
$$

Therefore $S$ is a solution of the following eigenvalue problem

$$
\left\{\begin{array}{l}
-g^{-1}(s) S^{\prime \prime}=\left(\lambda_{1}-\mu_{1}\right) S \text { in }(0, l) \\
S(0)=S^{\prime}(l)=0 .
\end{array}\right.
$$

By differentiating one obtains

$$
-n^{2} \omega_{n}^{\frac{2}{n}} s^{2-\frac{2}{n}} W^{\prime \prime}-n^{2} \omega_{n}^{\frac{2}{n}}\left(2-\frac{2}{n}\right) s^{1-\frac{2}{n}} W^{\prime}=\left(\lambda_{1}-\mu_{1}\right) W,
$$

where

$$
W(s)=S^{\prime}(s)
$$


If we set

$$
\begin{aligned}
s & =\omega_{n} r^{n} \\
R & =\left(\frac{l}{\omega_{n}}\right)^{\frac{1}{n}} \\
\widetilde{W}(r) & =W\left(\omega_{n} r^{n}\right),
\end{aligned}
$$

a straightforward calculation gives

$$
\left\{\begin{array}{l}
-\widetilde{W^{\prime \prime}}(r)-\frac{n-1}{r} \widetilde{W}^{\prime}(r)=\left(\lambda_{1}-\mu_{1}\right) \widetilde{W} \quad \text { in }(0, R) \\
\widetilde{W}(R)=\widetilde{W}^{\prime}(0)=0
\end{array}\right.
$$

Therefore

$$
\widetilde{W}(r)=r^{1-\frac{n}{2}} J_{\frac{n}{2}-1}\left(j \frac{n}{2}-1,1 \frac{r}{R}\right),
$$

where $R>0$ has to be chosen in such a way that $\left(\lambda_{1}-\mu_{1}\right)$ is the first eigenvalue of (3.10); this fact implies

$$
\begin{aligned}
\left(\lambda_{1}-\mu_{1}\right) & =\frac{j_{\frac{n}{2}-1,1}^{2}}{R^{2}} \\
R & =\frac{j_{\frac{n}{2}-1,1}}{\left(\lambda_{1}-\mu_{1}\right)^{1 / 2}} \\
l & =\omega_{n} R^{n}=\frac{\omega_{n} j_{\frac{n}{2}-1,1}^{n}}{\left(\lambda_{1}-\mu_{1}\right)^{n / 2}} .
\end{aligned}
$$

Finally

$$
\begin{aligned}
\widetilde{W}(r) & =r^{1-\frac{n}{2}} J_{\frac{n}{2}-1}\left(\left(\lambda_{1}-\mu_{1}\right)^{1 / 2} r\right) \\
W(s) & =\widetilde{W}\left(\left(\frac{s}{\omega_{n}}\right)^{1 / n}\right)=\left(\frac{s}{\omega_{n}}\right)^{\frac{2-n}{2 n}} J_{\frac{n}{2}-1}\left(\left(\lambda_{1}-\mu_{1}\right)^{1 / 2}\left(\frac{s}{\omega_{n}}\right)^{1 / n}\right) \\
S(s) & =\int_{0}^{s}\left(\frac{\sigma}{\omega_{n}}\right)^{\frac{2-n}{2 n}} J_{\frac{n}{2}-1}\left(\left(\lambda_{1}-\mu_{1}\right)^{1 / 2}\left(\frac{\sigma}{\omega_{n}}\right)^{1 / n}\right) d \sigma
\end{aligned}
$$

and the thesis follows.

At this point we want to introduce some functional spaces, naturally associated with problems of the type (3.3). 
Let $\Gamma$ be the portion of $\partial\left(I_{l} \times \Omega^{\prime \prime}\right)$ where the Dirichlet boundary condition is prescribed for $V$ in problem (3.3). Clearly, by (3.1), on the remaining part of the boundary, there is a Neumann condition imposed on $V$.

Let us consider the set of functions

$$
\mathcal{H}=\left\{\phi \in C^{\infty}\left(I_{l} \times \Omega^{\prime \prime}\right): \Gamma \cap \operatorname{supp} \phi=\emptyset\right\},
$$

and the following two norms

$$
\begin{aligned}
\|\phi\|^{2} & =\int_{I_{l} \times \Omega^{\prime \prime}}\left(\phi_{s}^{2}+\left|D_{y} \phi\right|^{2}\right) d s d y \\
\|\| \phi\|\|^{2} & =\int_{I_{l} \times \Omega^{\prime \prime}}\left(\phi_{s}^{2}+\left|D_{y} \phi\right|^{2} g+\phi^{2} g\right) d s d y .
\end{aligned}
$$

Finally, let us denote

$$
\begin{aligned}
H_{0, \Gamma}^{1}\left(I_{l} \times \Omega^{\prime \prime}\right) & =\overline{\mathcal{H}}^{\|\cdot \cdot\|} \\
H_{0, \Gamma}^{1}\left(I_{l} \times \Omega^{\prime \prime} ; g\right) & =\overline{\mathcal{H}}^{\|\cdot \cdot\| \|},
\end{aligned}
$$

and by $L^{2}\left(I_{l} \times \Omega^{\prime \prime} ; g\right)$, the set of those functions such that

$$
\int_{I_{l} \times \Omega^{\prime \prime}} \phi^{2} g d s d y<+\infty .
$$

Clearly, by the definition of $g, H_{0, \Gamma}^{1}\left(I_{l} \times \Omega^{\prime \prime} ; g\right)$ is continuously embedded in $H_{0, \Gamma}^{1}\left(I_{l} \times \Omega^{\prime \prime}\right)$.

The next two Lemmas show that a Poincarè inequality holds in $H_{0, \Gamma}^{1}\left(I_{l} \times \Omega^{\prime \prime} ; g\right)$ and, furthermore, the embedding of $H_{0, \Gamma}^{1}\left(I_{l} \times \Omega^{\prime \prime} ; g\right)$ into $L^{2}\left(I_{l} \times \Omega^{\prime \prime} ; g\right)$ is compact. In view of this results, the space $H_{0, \Gamma}^{1}\left(I_{l} \times\right.$ $\left.\Omega^{\prime \prime} ; g\right)$ will be equipped with the norm

$$
\left(\int_{I_{l} \times \Omega^{\prime \prime}}\left(\phi_{s}^{2}+\left|D_{y} \phi\right|^{2} g\right) d s d y\right)^{1 / 2} .
$$

Lemma 2. It holds that

$$
\int_{I_{l} \times \Omega^{\prime \prime}} \phi^{2} g d y d s \leq \mu_{1} \int_{I_{l} \times \Omega^{\prime \prime}}\left|D_{y} \phi\right|^{2} g d y d s, \quad \forall \phi \in H_{0, \Gamma}^{1}\left(I_{l} \times \Omega^{\prime \prime} ; g\right),
$$

where $\mu_{1}$ is the first eigenvalue of the problem (3.6).

Proof: For any $\phi$ in $\mathcal{H}$ it happens

$$
\int_{\Omega^{\prime \prime}} \phi^{2}(s, y) d y \leq \mu_{1} \int_{\Omega^{\prime \prime}}\left|D_{y} \phi\right|^{2} d y, \quad \forall s \in I_{l},
$$


therefore

$$
g(s) \int_{\Omega^{\prime \prime}} \phi^{2}(s, y) d y \leq \mu_{1} g(s) \int_{\Omega^{\prime \prime}}\left|D_{y} \phi\right|^{2} d y, \quad \forall s \in I_{l},
$$

and thus

$$
\int_{I_{l}}\left(g(s) \int_{\Omega^{\prime \prime}} \phi^{2}(s, y) d y\right) d s \leq \mu_{1} \int_{I_{l}}\left(g(s) \int_{\Omega^{\prime \prime}}\left|D_{y} \phi\right|^{2} d y\right) d s .
$$

By density the claim follows.

Lemma 3. The embedding of $H_{0, \Gamma}^{1}\left(I_{l} \times \Omega^{\prime \prime} ; g\right)$ into $L^{2}\left(I_{l} \times \Omega^{\prime \prime} ; g\right)$ is compact.

Proof: Our aim is to show that if $\phi_{k}$ is a bounded sequence in $H_{0, \Gamma}^{1}\left(I_{l} \times\right.$ $\left.\Omega^{\prime \prime} ; g\right)$ i.e.

$$
\int_{I_{l} \times \Omega^{\prime \prime}}\left(\left|D_{y} \phi_{k}\right|^{2} g+\left(\phi_{k}\right)_{s}^{2}\right) d s d y \leq C
$$

then, up to a subsequence, it holds that

$$
\int_{I_{l} \times \Omega^{\prime \prime}}\left(\phi_{k}-\phi\right)^{2} g d s d y \rightarrow 0,
$$

for some $\phi \in H_{0, \Gamma}^{1}\left(I_{l} \times \Omega^{\prime \prime} ; g\right)$.

We observe that, Hardy inequality (2.2), together with Fubini Theorem, implies

$$
\int_{I_{l} \times \Omega^{\prime \prime}} \frac{\phi^{2}}{s^{2}} d s d y \leq \frac{1}{4} \int_{I_{l} \times \Omega^{\prime \prime}} \phi_{s}^{2} d s d y, \quad \forall \phi \in \mathcal{H},
$$

and, a fortiori, one has

$$
\int_{I_{l} \times \Omega^{\prime \prime}} \frac{\phi^{2}}{s^{2}} d s d y \leq \frac{1}{4} \int_{I_{l} \times \Omega^{\prime \prime}}\left(\left|D_{y} \phi\right|^{2} g+\phi_{s}^{2}\right) d s d y, \quad \forall \phi \in \mathcal{H} .
$$

By density, the inequality above holds for any $\phi$ in $H_{0, \Gamma}^{1}\left(I_{l} \times \Omega^{\prime \prime} ; g\right)$.

Clearly, by classical functional analysis, there exists $\widetilde{\phi} \in H_{0, \Gamma}^{1}\left(I_{l} \times\right.$ $\left.\Omega^{\prime \prime} ; g\right)$, such that, up to a subsequence,

$$
\phi_{k} \rightarrow \widetilde{\phi} \text { weakly in } H_{0, \Gamma}^{1}\left(I_{l} \times \Omega^{\prime \prime} ; g\right) \text {. }
$$

The fact that $H_{0, \Gamma}^{1}\left(I_{l} \times \Omega^{\prime \prime} ; g\right)$ is continuously embedded in $H_{0, \Gamma}^{1}\left(I_{l} \times \Omega^{\prime \prime}\right)$, together with (3.12), ensures that, modulo a subsequence,

$$
\phi_{k} \rightarrow \widetilde{\phi}\left\{\begin{array}{l}
\text { a.e. in } I_{l} \times \Omega^{\prime \prime} \\
\text { strongly in } L^{q}\left(I_{l} \times \Omega^{\prime \prime}\right), \quad \forall q \in\left[1,2^{*}\right) \\
\text { weakly in } H_{0, \Gamma}^{1}\left(I_{l} \times \Omega^{\prime \prime}\right),
\end{array}\right.
$$


where

$$
2^{*}= \begin{cases}+\infty, & \text { if } m=1 \\ \frac{2(m+1)}{m-1}, & \text { if } m \geq 2 .\end{cases}
$$

Now let us prove (3.13) with $\phi=\widetilde{\phi}$. For any given $\epsilon$ in $(0, l)$, since $\phi_{k}$ strongly converges to $\widetilde{\phi}$ in $L^{2}\left(I_{l} \times \Omega^{\prime \prime}\right)$, there exists $k_{\epsilon} \in \mathbb{N}$ such that

$$
\int_{(0, l) \times \Omega^{\prime \prime}}\left(\phi_{k}-\widetilde{\phi}\right)^{2} d s d y \leq \epsilon^{2}, \quad \forall k \geq k_{\epsilon} .
$$

Hence, for any $k \geq k_{\epsilon}$, by (3.14), (3.12) and (3.16), we get

$$
\begin{aligned}
\int_{(0, l) \times \Omega^{\prime \prime}}\left(\phi_{k}-\widetilde{\phi}\right)^{2} g d s d y= & n^{-2} \omega_{n}^{-\frac{2}{n}} \int_{(0, \epsilon) \times \Omega^{\prime \prime}} \frac{\left(\phi_{k}-\widetilde{\phi}\right)^{2}}{s^{2}} s^{\frac{2}{N}} d s d y \\
& +n^{-2} \omega_{n}^{-\frac{2}{n}} \int_{(\epsilon, l) \times \Omega^{\prime \prime}} \frac{\left(\phi_{k}-\widetilde{\phi}\right)^{2}}{s^{2-\frac{2}{n}}} d s d y \\
\leq & \epsilon^{\frac{2}{n}} n^{-2} \omega_{n}^{-\frac{2}{n}} \int_{(0, \epsilon) \times \Omega^{\prime \prime}} \frac{\left(\phi_{k}-\widetilde{\phi}\right)^{2}}{s^{2}} d s d y \\
& +\frac{n^{-2} \omega_{n}^{-\frac{2}{n}}}{\epsilon^{2-\frac{2}{n}}} \int_{(\epsilon, l) \times \Omega^{\prime \prime}}\left(\phi_{k}-\widetilde{\phi}\right)^{2} d s d y \\
\leq & C \epsilon^{\frac{2}{n}},
\end{aligned}
$$

that is $(3.13)$.

Remark 1 . The above lemmas are trivial when $n=1$, since, in that case, the function $g(s)$ is a constant.

Now we are in position to state the main result of this section.

Theorem 3. It is possible to normalize $u$ and $v$ in such a way to have

$$
\int_{0}^{l} u^{*}(\sigma, y) d \sigma \leq \int_{0}^{l} v^{*}(\sigma, y) d \sigma, \quad \forall y \in \Omega^{\prime \prime},
$$

moreover, once (3.17) is fulfilled, then

$$
\int_{0}^{s} u^{*}(\sigma, y) d \sigma \leq \int_{0}^{s} v^{*}(\sigma, y) d \sigma, \quad \forall(s, y) \in I_{l} \times \Omega^{\prime \prime} .
$$


58

F. CHIACCHiO

Proof: From Lemma 1, recalling the definition of $V$ given in (3.5) and (3.3), we know that

(3.19) $\begin{cases}-\Delta_{y}\left(\int_{0}^{l} v^{*}(\sigma, y) d \sigma\right)=\mu_{1}\left(\int_{0}^{l} v^{*}(\sigma, y) d \sigma\right) & \text { in } \Omega^{\prime \prime} \\ \int_{0}^{l} v^{*}(\sigma, y) d \sigma=0 & \text { on } \partial \Omega^{\prime \prime} .\end{cases}$

Since $\Omega^{\prime \prime}$ is smooth, from the Hop maximum principle, we deduce

$$
\left|D_{y}\left(\int_{0}^{l} v^{*}(\sigma, y) d \sigma\right)\right| \geq C, \quad \forall y \in \partial \Omega^{\prime \prime},
$$

for some $C>0$.

Now we claim that $\int_{0}^{l} u^{*}(\sigma, y) d \sigma$ is uniformly Lipschitz continuous up to $\partial \Omega^{\prime \prime}$, this statement, together with (3.20), will ensure (3.17).

Inequality (3.2) gives

$$
\begin{array}{r}
-\left.\frac{\partial u^{*}}{\partial s}\right|_{s=l}-g(l) \Delta_{y}\left(\int_{0}^{l} u^{*}(\sigma, y) d \sigma\right) \\
\quad \leq \lambda_{1} g(l)\left(\int_{0}^{l} u^{*}(\sigma, y) d \sigma\right) \text { in } \Omega^{\prime \prime}
\end{array}
$$

and, since

$$
-\left.\frac{\partial u^{*}}{\partial s}\right|_{s=l} \geq 0
$$

we deduce

$$
\begin{cases}-\Delta_{y}\left(\int_{0}^{l} u^{*}(\sigma, y) d \sigma\right) \leq \lambda_{1} \int_{0}^{l} u^{*}(\sigma, y) d \sigma & \text { in } \Omega^{\prime \prime} \\ \int_{0}^{l} u^{*}(\sigma, y) d \sigma=0 & \text { on } \partial \Omega^{\prime \prime} .\end{cases}
$$

Now let us consider the solution of the problem

$$
\begin{cases}-\Delta_{y} \psi=M & \text { in } \Omega^{\prime \prime} \\ \psi=0 & \text { on } \partial \Omega^{\prime \prime}\end{cases}
$$

where

$$
M=\lambda_{1} \max _{y \in \Omega^{\prime \prime}} \int_{0}^{l} u^{*}(\sigma, y) d \sigma>0 .
$$


Since $\Omega^{\prime \prime}$ is $C^{2, \alpha}$, the function $\psi$, see [14], is uniformly Lipschitz continuous up to $\partial \Omega^{\prime \prime}$, and moreover

$$
0 \leq \int_{0}^{l} u^{*}(\sigma, y) d \sigma \leq \psi(y) \text { in } \overline{\Omega^{\prime \prime}} .
$$

This last inequality ensures the claim that $\int_{0}^{l} u^{*}(\sigma, y) d \sigma$ is uniformly Lipschitz continuous up to $\partial \Omega^{\prime \prime}$.

Let us consider the following problem

$$
\left\{\begin{array}{l}
-\Phi_{s s}-g(s) \Delta_{y} \Phi=\lambda g(s) \Phi \\
\Phi \in H_{0, \Gamma}^{1}\left(I_{l} \times \Omega^{\prime \prime} ; g\right) .
\end{array}\right.
$$

By Lemma 3 the differential operator appearing in (3.25) is selfadjoint and compact (as an application from $L^{2}\left(I_{l} \times \Omega^{\prime \prime} ; g\right)$ onto $L^{2}\left(I_{l} \times \Omega^{\prime \prime} ; g\right)$ ). As it is well-known, the first eigenvalue of problem (3.25) is the minimum of the Rayleigh quotient

$R(\Psi)=\frac{\int_{I_{l} \times \Omega^{\prime \prime}}\left(\Psi_{s}^{2}+g(s)\left|D_{y} \Psi\right|^{2}\right) d s d y}{\int_{I_{l} \times \Omega^{\prime \prime}} \Psi^{2} g d s d y}$, with $\Psi \in H_{0, \Gamma}^{1}\left(I_{l} \times \Omega^{\prime \prime} ; g\right)-\{0\}$.

The corresponding eigenfunction satisfies in $I_{l} \times \Omega^{\prime \prime}$ an Harnack inequality and therefore it has one sign within $\Omega$. As a consequence the first eigenvalue of (3.25) is simple. Now we observe that the function $V(s, y)=\int_{0}^{s} v^{*}(\sigma, y) d \sigma$ verifies

$$
\left\{\begin{array}{l}
-V_{s s}-g(s) \Delta_{y} V=\lambda_{1} g(s) V \\
V \in H_{0, \Gamma}^{1}\left(I_{l} \times \Omega^{\prime \prime} ; g\right),
\end{array}\right.
$$

by definition

$$
V(s, y)>0 \text { in } I_{l} \times \Omega^{\prime \prime},
$$

then we conclude that

$$
\min _{\Psi \in H_{0, \Gamma}^{1}\left(I_{l} \times \Omega^{\prime \prime} ; g\right)-\{0\}} R(\Psi)=R(V)=\lambda_{1} .
$$

If $l=L$, functions $U$ and $V$ are easily verified to be proportional. If, instead, $l<L$, then

$$
\frac{\partial U}{\partial s}(l, y)=u^{*}(l, y)>0, \quad \forall y \in \Omega^{\prime \prime},
$$

and

$$
\frac{\partial V}{\partial s}(l, y)=v^{*}(l, y)=0, \quad \forall y \in \Omega^{\prime \prime}
$$


Ab absurdo, we suppose that the set

$$
A_{+}=\left\{(s, y) \in I_{l} \times \Omega^{\prime \prime}: U(s, y)-V(s, y)>0\right\}
$$

in nonempty. Then, by (3.17), it holds

$$
Z(s, y) \equiv U(s, y)-V(s, y)=0 \text { on } \partial A_{+} \cdot
$$

By setting $Z=0$ outside $A_{+}$, from (3.2) and (3.3), one deduces that

$$
R(Z) \leq \lambda_{1}
$$

This implies that $Z$ is an eigenfunction corresponding to $\lambda_{1}$, but, since, $\lambda_{1}$ is simple, there exists a constant $C \neq 0$ such that

$$
Z(s, y)=C V(s, y) \text { in } I_{l} \times \Omega^{\prime \prime}
$$

i.e.

$$
U(s, y)=(C+1) V(s, y) \text { in } I_{l} \times \Omega^{\prime \prime} .
$$

We can conclude that

$$
\left.\frac{\partial U}{\partial s}\right|_{s=l}=\left.(C+1) \frac{\partial V}{\partial s}\right|_{s=l}=0
$$

which contradicts $(3.26)$.

\section{More general differential operators}

Let $\Omega$ be as in the previous section, and let us consider the following class of operators

(4.1) $\quad A: w \in H_{0}^{1}(\Omega) \rightarrow\left\{-\left(a_{i j}(x, y) w_{x_{i}}\right)_{x_{j}}-\left(b_{h k}(y) w_{y_{h}}\right)_{y_{k}}\right\} \in H^{-1}$

where

i) $A$ is symmetric, i.e.

$$
a_{i j}(x, y)=a_{j i}(x, y) \text { and } b_{h k}(y)=b_{k h}(y), \text { a.e. }(x, y) \in \Omega,
$$

ii) $A$ satisfies the following ellipticity assumption

$$
|\xi|^{2}+\nu|\eta|^{2} \leq a_{i j}(x, y) \xi_{i} \xi_{j}+b_{h k}(y) \eta_{h} \eta_{k} \leq \Theta\left(|\xi|^{2}+\nu|\eta|^{2}\right),
$$

for some $\nu>0$ and $\Theta>1, \forall(\xi, \eta) \in \mathbb{R}^{n} \times \mathbb{R}^{m}$ and a.e. $(x, y) \in \Omega$,

iii) coefficients $b_{k h}(y)$ are analytic in $\overline{\Omega^{\prime \prime}}$.

Let $\lambda_{1}$ be the first eigenvalue of the problem

$$
\left\{\begin{array}{l}
A w=\lambda w \\
w \in H_{0}^{1}(\Omega),
\end{array}\right.
$$


and let $u_{1}$ be the corresponding eigenfunction, normalized as follows

$$
\left\{\begin{array}{l}
\int_{\Omega} u_{1}^{2} d x d y=1 \\
u_{1}>0
\end{array} \text { in } \Omega .\right.
$$

Clearly

$$
\lambda_{1}=\min _{w \in H_{0}^{1}(\Omega)-\{0\}} \frac{\langle A w, w\rangle}{\int_{\Omega} w^{2} d x d y}=\left\langle A u_{1}, u_{1}\right\rangle .
$$

Now we want to estimate $u_{1}$ in terms of the first eigenfunction of the following "symmetrized" problem

$$
\left\{\begin{array}{l}
A^{\sharp} v \equiv-\Delta_{x} v-\left(b_{h k}(y) v_{y_{h}}\right)_{y_{k}}=\lambda v \\
v \in H_{0}^{1}\left(B_{R} \times \Omega^{\prime \prime}\right),
\end{array}\right.
$$

where again we choose $R$ in such a way that the first eigenvalue of (4.5) coincides with $\lambda_{1}$. Let us denote

$$
l=\left|B_{R}\right|_{n} .
$$

For our purposes we need a Faber-Krahn type inequality, which will be an easy consequence of the following Pólya-Szegö type principle.

Lemma 4. It holds that

$$
\langle A u, u\rangle \geq\left\langle A^{\sharp} u^{\sharp}, u^{\sharp}\right\rangle, \quad \forall u \in H_{0}^{1}(\Omega) .
$$

Proof: In this lemma, all the functions involved will be defined in the whole $\mathbb{R}^{n+m}$, by setting zero their value outside $\Omega$ or $\Omega^{\sharp}$. By the ellipticity condition (4.3) and Pólya-Szegö principle (2.1) we have that

$$
\begin{aligned}
\langle A u, u\rangle & \geq \int_{\mathbb{R}^{n+m}}\left|D_{x} u\right|^{2} d x d y+\int_{\mathbb{R}^{n+m}} b_{h k}(y) \frac{\partial u}{\partial y_{h}} \frac{\partial u}{\partial y_{k}} d x d y \\
& \geq \int_{\mathbb{R}^{n+m}}\left|D_{x} u^{\sharp}\right|^{2} d x d y+\int_{\mathbb{R}^{n+m}} b_{h k}(y) \frac{\partial u}{\partial y_{h}} \frac{\partial u}{\partial y_{k}} d x d y .
\end{aligned}
$$

We are done once we show that

$$
\int_{\mathbb{R}^{n+m}} b_{h k}(y) \frac{\partial u}{\partial y_{h}} \frac{\partial u}{\partial y_{k}} d x d y \geq \int_{\mathbb{R}^{n+m}} b_{h k}(y) \frac{\partial u^{\sharp}}{\partial y_{h}} \frac{\partial u^{\sharp}}{\partial y_{k}} d x d y .
$$

Inequality above is easily proved when the matrix $b_{h k}(y)$ is diagonal. In that case, indeed, by (4.3) one has $b_{h h}(y) \geq \nu>0$ in $\mathbb{R}^{m}$, and we have (see for instance Lemma 5.1 of [9] )

$$
\int_{\mathbb{R}^{n}}\left(\frac{\partial u}{\partial y_{h}}\right)^{2} d x \geq \int_{\mathbb{R}^{n}}\left(\frac{\partial u^{\sharp}}{\partial y_{h}}\right)^{2} d x, \text { a.e. } y \in \mathbb{R}^{m} \text { and } \forall h \in\{1, \ldots, m\}
$$


and clearly

$$
\begin{array}{r}
\text { (4.8) } b_{h h}(y) \int_{\mathbb{R}^{n}}\left(\frac{\partial u}{\partial y_{h}}\right)^{2} d x \geq b_{h h}(y) \int_{\mathbb{R}^{n}}\left(\frac{\partial u^{\sharp}}{\partial y_{h}}\right)^{2} d x, \\
\text { a.e. } y \in \mathbb{R}^{m} \text { and } \forall h \in\{1, \ldots, m\} .
\end{array}
$$

Finally summing on $h$ and integrating over $\mathbb{R}^{m}$ one gets the claim. Note also that, if $E$ is any measurable subset of $\mathbb{R}^{m}$, then (4.8) immediately implies that

$$
\begin{array}{r}
\int_{E \times \mathbb{R}^{n}} b_{h h}(y)\left(\frac{\partial u}{\partial y_{h}}\right)^{2} d x d y \geq \int_{E \times \mathbb{R}^{n}} b_{h h}(y)\left(\frac{\partial u^{\sharp}}{\partial y_{h}}\right)^{2} d x d y, \\
\forall h \in\{1, . ., m\} .
\end{array}
$$

Now, in order to reduce ourselves to the diagonal case, we argue by approximation. For any $\epsilon>0$, one can find a sequence of simple functions

$$
b_{h k}^{\epsilon}(y)=\sum_{p=1}^{\infty}\left(\widetilde{b}_{h k}\right)^{\epsilon, p} \chi_{E^{\epsilon, p}},
$$

where $E^{\epsilon, p}$ are measurable and mutually disjoint subsets of $\mathbb{R}^{m}$ such that

$$
\bigcup_{p=1}^{\infty} E^{\epsilon, p}=\mathbb{R}^{m}, \quad \forall \epsilon>0,
$$

and

$$
\begin{cases}\left(\widetilde{b}_{h k}\right)^{\epsilon, p}=\left(\widetilde{b}_{k h}\right)^{\epsilon, p} & \\ \sup _{y \in \mathbb{R}^{m}}\left|b_{h k}^{\epsilon}(y)-b_{h k}(y)\right|=o(1), & \text { as } \epsilon \rightarrow 0^{+} \\ b_{h k}^{\epsilon}(y) \eta_{h} \eta_{k} \geq \nu_{\epsilon}|\eta|^{2}=(\nu+o(1))|\eta|^{2}, & \forall \eta \in \mathbb{R}^{m} .\end{cases}
$$

At this point we have

$$
\begin{aligned}
\int_{\mathbb{R}^{n+m}} b_{h k}^{\epsilon}(y) \frac{\partial u}{\partial y_{h}} \frac{\partial u}{\partial y_{k}} d x d y & =\int_{\mathbb{R}^{n}}\left(\int_{\mathbb{R}^{m}} b_{h k}^{\epsilon}(y) \frac{\partial u}{\partial y_{h}} \frac{\partial u}{\partial y_{k}} d y\right) d x \\
& =\int_{\mathbb{R}^{n}}\left(\sum_{p=1}^{\infty} \int_{E^{\epsilon, p}}\left(\widetilde{b}_{h k}\right)^{\epsilon, p} \frac{\partial u}{\partial y_{h}} \frac{\partial u}{\partial y_{k}} d y\right) d x
\end{aligned}
$$

In each set $E^{\epsilon, p}$ the integral can be carried out over a set of coordinates $\left(y_{1}^{\epsilon, p}, \ldots, y_{m}^{\epsilon, p}\right)$ which diagonalizes the matrix $B^{\epsilon, p}$ of entries $\left(\widetilde{b}_{h k}\right)^{\epsilon, p}$. Hence, for each $\epsilon$ and $p$, there exists an orthogonal matrix $T^{\epsilon, p}$ such that

$$
y^{\epsilon, p}=T^{\epsilon, p} y,
$$


and

$$
T^{\epsilon, p} B^{\epsilon, p}\left(T^{\epsilon, p}\right)^{-1}=D^{\epsilon, p},
$$

where $D^{\epsilon, p}$ is a diagonal matrix whose entries will be denoted with $d_{h, k}^{\epsilon, p}$.

Note that

$$
d_{h, h}^{\epsilon, p} \geq \nu+o(1), \quad \forall p \in \mathbb{N} \text { and } \forall h \in\{1, \ldots, m\},
$$

so for $\epsilon$ small enough, say $\epsilon \in\left(0, \epsilon_{0}\right)$, it holds

$$
d_{h, h}^{\epsilon, p} \geq \frac{\nu}{2}>0, \quad \forall p \in \mathbb{N} \text { and } \forall h \in\{1, \ldots, m\} .
$$

By performing the above change of variables in each set $E^{\epsilon, p}$, we get

$$
\begin{aligned}
& \int_{\mathbb{R}^{n}}\left(\int_{\mathbb{R}^{m}} b_{h k}^{\epsilon}(y) \frac{\partial u}{\partial y_{h}} \frac{\partial u}{\partial y_{k}} d y\right) d x \\
= & \int_{\mathbb{R}^{n}}\left(\sum_{p=1}^{\infty} \int_{E^{\epsilon, p}}\left(\widetilde{b}_{h k}\right)^{\epsilon, p} \frac{\partial u}{\partial y_{h}} \frac{\partial u}{\partial y_{k}} d y\right) d x \\
= & \int_{\mathbb{R}^{n}}\left(\sum_{p=1}^{\infty} \int_{\left(T^{\epsilon, p}\right)^{-1} E^{\epsilon, p}}\left(d_{h h}\right)^{\epsilon, p} \frac{\partial u}{\partial y_{h}^{\epsilon, p}} \frac{\partial u}{\partial y_{h}^{\epsilon, p}} d y^{\epsilon, p}\right) d x .
\end{aligned}
$$

For $\epsilon \in\left(0, \epsilon_{0}\right)$, using (4.9) and finally coming back to the old variables, we conclude

$$
\begin{aligned}
& \int_{\mathbb{R}^{n}}\left(\sum_{p=1}^{\infty} \int_{\left(T^{\epsilon, p}\right)^{-1} E^{\epsilon, p}}\left(d_{h h}\right)^{\epsilon, p} \frac{\partial u}{\partial y_{h}^{\epsilon, p}} \frac{\partial u}{\partial y_{h}^{\epsilon, p}} d y^{\epsilon, p}\right) d x \\
\geq & \int_{\mathbb{R}^{n}}\left(\sum_{p=1}^{\infty} \int_{\left(T^{\epsilon, p}\right)^{-1} E^{\epsilon, p}}\left(d_{h h}\right)^{\epsilon, p} \frac{\partial u^{\sharp}}{\partial y_{h}^{\epsilon, p}} \frac{\partial u^{\sharp}}{\partial y_{h}^{\epsilon, p}} d y^{\epsilon, p}\right) d x \\
= & \int_{\mathbb{R}^{n}}\left(\sum_{p=1}^{\infty} \int_{E^{\epsilon, p}}\left(\widetilde{b}_{h k}\right)^{\epsilon, p} \frac{\partial u^{\sharp}}{\partial y_{h}} \frac{\partial u^{\sharp}}{\partial y_{k}} d y\right) d x \\
= & \int_{\mathbb{R}^{n}}\left(\int_{\mathbb{R}^{m}} b_{h k}^{\epsilon}(y) \frac{\partial u^{\sharp}}{\partial y_{h}} \frac{\partial u^{\sharp}}{\partial y_{k}} d y\right) d x .
\end{aligned}
$$

Passing to the limit as $\epsilon$ goes to $0^{+}$, we get (4.7) and therefore (4.6).

Observing the proof, is clear that the above result still holds true assuming that $b_{h k}(y)$ are just in $C^{0}\left(\overline{\Omega^{\prime \prime}}\right)$.

As an immediate consequence of Lemma 4 , is the following FaberKrahn inequality. 
Lemma 5. It holds that

$$
\begin{aligned}
\lambda_{1}(\Omega) & =\min _{u \in H_{0}^{1}(\Omega)-\{0\}} \frac{\langle A u, u\rangle}{\int_{\Omega} u^{2} d x d y} \\
& \geq \min _{v \in H_{0}^{1}\left(\Omega^{\sharp}\right)-\{0\}} \frac{\left\langle A^{\sharp} v, v\right\rangle}{\int_{\Omega^{\sharp}} v^{2} d x d y}=\lambda_{1}\left(\Omega^{\sharp}\right),
\end{aligned}
$$

and therefore

$$
l \leq L
$$

where $L$ is defined in (3.1).

Since the coefficients of $A^{\sharp}$ are all analytic, the already mentioned results in $[\mathbf{2}]$, allow to conclude that

$$
\begin{cases}-V_{s s}-g(s)\left(b_{h k}(y) V_{y_{h}}\right)_{y_{k}}=\lambda_{1} g(s) V & \text { in } I_{l} \times \Omega^{\prime \prime} \\ V(0, y)=V_{s}(l, y)=0 & \forall y \in \Omega^{\prime \prime} \\ V(s, y)=0 & \forall(s, y) \in I_{l} \times \partial \Omega^{\prime \prime}\end{cases}
$$

where

$$
V(s, y)=\int_{0}^{s} v^{*}(\sigma, y) d \sigma, \quad(s, y) \in I_{l} \times \Omega^{\prime \prime} .
$$

For our purposes it will be useful to rewrite (4.12) in its weak form. To this aim, we consider the following problem

$$
\left\{\begin{array}{l}
-\Phi_{s s}-\left(g(s) b_{h k}(y) \Phi_{y_{h}}\right)_{y_{k}}=\lambda g(s) \Phi \\
\Phi \in H_{0, \Gamma}^{1}\left(I_{l} \times \Omega^{\prime \prime} ; g\right) .
\end{array}\right.
$$

Arguing as in Theorem 3 we can still say that the differential operator appearing in (4.14) is selfadjoint and compact, that the first eigenvalue is simple and that a corresponding eigenfunction does not change its sign in $I_{l} \times \Omega^{\prime \prime}$. Moreover the first eigenvalue realizes the minimum

$$
\min _{\Psi \in H_{0, \Gamma}^{1}\left(I_{l} \times \Omega^{\prime \prime} ; g\right)-\{0\}} \frac{\int_{I_{l} \times \Omega^{\prime \prime}}\left(\Psi_{s}^{2}+g(s) b_{h k}(y) \Psi_{y_{h}} \Psi_{y_{k}}\right) d s d y}{\int_{I_{l} \times \Omega^{\prime \prime}} \Psi^{2} g d s d y} .
$$

The analogous of (3.8) and (3.9) still holds true. To this aim, let $\mu_{1}$ be the first eigenvalue of the problem

$$
\left\{\begin{array}{l}
-\left(b_{h k}(y) w_{y_{h}}\right)_{y_{k}}=\mu w \quad \text { in } \Omega^{\prime \prime} \\
w \in H_{0}^{1}\left(\Omega^{\prime \prime}\right)
\end{array}\right.
$$


and $\chi(y)$ a corresponding eigenfunction. Repeating the arguments used for Lemma 1, we get

Lemma 6. We have that the function $V(s, y)$, defined in (4.12), is proportional to

$$
\begin{gathered}
\chi(y) \int_{0}^{s}\left(\frac{\sigma}{\omega_{n}}\right)^{\frac{2-n}{2 n}} J_{\frac{n}{2}-1}\left(\left(\lambda_{1}-\mu_{1}\right)^{1 / 2}\left(\frac{\sigma}{\omega_{n}}\right)^{1 / n}\right) d \sigma \\
l=\frac{\omega_{n} j_{\frac{n}{2}-1,1}^{2}}{\left(\lambda_{1}-\mu_{1}\right)^{n / 2}}
\end{gathered}
$$

and, finally, $V(s, y)$ is the first eigenfunction of (4.14).

Since some coefficients of the operator $A$ are just bounded, we can not apply directly the results in [2] in order to get an inequality analogous to (3.2). We overcome the lack of regularity by means of the following approximation procedure.

Firstly we define the coefficients $a_{i j}(x, y)$ on the whole $\mathbb{R}^{n+m}$ as follows

$$
a_{i j}(x, y)= \begin{cases}a_{i j}(x, y) & \text { if }(x, y) \in \Omega \\ \delta_{i j} & \text { if }(x, y) \in \mathbb{R}^{n+m} \backslash \Omega .\end{cases}
$$

Finally, we will denote by $a_{i j}^{\epsilon}(x, y)$ the convolution of such functions with the Heat kernel $\rho^{\epsilon}$, where

$$
\rho^{\epsilon}(x, y)=\frac{1}{(4 \pi \epsilon)^{\frac{n+m}{2}}} \exp \left(-\frac{|x|^{2}+|y|^{2}}{4 \epsilon}\right) \text {, with } \epsilon>0 .
$$

Note that, for each $\epsilon>0$, the functions $a_{i j}^{\epsilon}(x, y)$ are analytic in $\mathbb{R}^{n+m}$ and they satisfy, together with $b_{h k}(y)$, conditions (4.2) and (4.3).

Lemma 7. Let us introduce the following sequence of operators

$$
A^{\epsilon}: w \in H_{0}^{1}(\Omega) \rightarrow\left\{-\left(a_{i j}^{\epsilon}(x, y) w_{x_{i}}\right)_{x_{j}}-\left(b_{h k}(y) w_{y_{h}}\right)_{y_{k}}\right\} \in H^{-1} .
$$

For any $\epsilon>0$, denote by $\lambda_{1}^{\epsilon}$ and $u_{1}^{\epsilon}$ the first eigenvalue and the corresponding eigenfunction of the problem

$$
\left\{\begin{array}{l}
A^{\epsilon} w=\lambda w \\
w \in H_{0}^{1}(\Omega)
\end{array}\right.
$$

with

$$
\int_{\Omega}\left(u_{1}^{\epsilon}\right)^{2} d x d y=1
$$


and

$$
u_{1}^{\epsilon}>0 \text { in } \Omega \text {. }
$$

Then, up to a subsequence, as $\epsilon$ goes to $0^{+}$,

$$
\left\{\begin{array}{l}
\lambda_{1}^{\epsilon} \rightarrow \lambda_{1} \\
u_{1}^{\epsilon} \rightarrow u_{1} \quad \text { uniformly in } \bar{\Omega} .
\end{array}\right.
$$

Proof: As it is immediate to check, $A^{\epsilon}$ verifies (4.2) and (4.3); moreover

$$
\lambda_{1}^{\epsilon}=\min _{w \in H_{0}^{1}(\Omega)-\{0\}} \frac{\left\langle A^{\epsilon} w, w\right\rangle}{\int_{\Omega} w^{2} d x d y}=\left\langle A^{\epsilon} u_{1}^{\epsilon}, u_{1}^{\epsilon}\right\rangle .
$$

The sequence $\lambda_{1}^{\epsilon}$ is bounded, indeed by (4.3)

$$
0<\lambda_{1}^{\epsilon} \leq\left\langle A^{\epsilon} u_{1}, u_{1}\right\rangle \leq \Theta \int_{\Omega}\left(\left|D_{x} u_{1}\right|^{2}+\nu\left|D_{y} u_{1}\right|^{2}\right) d x d y \leq C,
$$

and therefore, along a subsequence, $\lambda_{1}^{\epsilon}$ goes to some $\widehat{\lambda} \in[0,+\infty)$.

On the other hand

$$
\int_{\Omega}\left(\left|D_{x} u_{1}^{\epsilon}\right|^{2}+\nu\left|D_{y} u_{1}^{\epsilon}\right|^{2}\right) d x d y \leq\left\langle A^{\epsilon} u_{1}^{\epsilon}, u_{1}^{\epsilon}\right\rangle=\lambda_{1}^{\epsilon} \leq C,
$$

and therefore there exists a function $\widehat{u} \in H_{0}^{1}(\Omega)$ such that

$$
u_{1}^{\epsilon} \rightarrow \widehat{u}\left\{\begin{array}{l}
\text { weakly in } H_{0}^{1}(\Omega) \\
\text { strongly in } L^{q}(\Omega), \quad \forall q \in\left[1,2^{*}\right) \\
\text { a.e. in } \Omega,
\end{array}\right.
$$

where now, with an abuse of notation, $2^{*}$ is defined as follows

$$
2^{*}= \begin{cases}+\infty, & \text { if } n+m=2 \\ \frac{2(n+m)}{n+m-2}, & \text { if } n+m \geq 3 .\end{cases}
$$

Now, passing to the limit as $\epsilon$ goes to $0^{+}$, in the weak formulation of $A^{\epsilon} u_{1}^{\epsilon}=\lambda_{1}^{\epsilon} u_{1}^{\epsilon}$

$$
\begin{array}{r}
\int_{\Omega}\left[a_{i j}^{\epsilon}(x, y) u_{1 x_{i}}^{\epsilon} \phi_{x_{j}}-b_{h k}(y) u_{1 y_{h}}^{\epsilon} \phi_{y_{k}}\right] d x d y=\lambda_{1}^{\epsilon} \int_{\Omega} u_{1}^{\epsilon} \phi d x d y \\
\forall \phi \in C_{0}^{\infty}(\Omega),
\end{array}
$$

one obtains that

$$
A \widehat{u}=\widehat{\lambda} \widehat{u}
$$


Observe that (4.17), (4.18) and (4.19) imply

$$
\begin{cases}\widehat{u} \geq 0 & \text { in } \Omega \\ \int_{\Omega} \widehat{u}^{2} d x d y=1 & \end{cases}
$$

and, obviously, $\widehat{u}$ cannot be identically zero. We claim that $\widehat{\lambda}=\lambda_{1}$. Indeed, if this is not the case, one would have

$$
\int_{\Omega} \widehat{u} u_{1} d x d y=0
$$

an absurd by the previous considerations. The fact that $\lambda_{1}$ is simple, in view of (4.4) and (4.20), guarantees that $\widehat{u}=u_{1}$.

Now it remains to show that the convergence of $u_{1}^{\epsilon}$ is uniform. The reverse Hölder inequality (see $[\mathbf{1 0}]$ ) ensures that

$$
\left\|u_{1}^{\epsilon}\right\|_{q} \leq C(q)\left\|u_{1}^{\epsilon}\right\|_{2}=C(q), \text { for any } q>2,
$$

and therefore, by standard elliptic estimates (see [14] for instance), the functions $u_{1}^{\epsilon}$ are equicontinuous and equibounded in $\bar{\Omega}$. Finally, the claim follows by a direct application of Ascoli-Arzelà Theorem.

Now we can state the comparison result in its full generality.

Theorem 4. It is possible to normalize $u$ and $v$ in such a way to have

$$
\int_{0}^{l} u^{*}(\sigma, y) d \sigma \leq \int_{0}^{l} v^{*}(\sigma, y) d \sigma, \quad \forall y \in \Omega^{\prime \prime},
$$

moreover, once (4.21) is fulfilled, then

$$
\int_{0}^{s} u^{*}(\sigma, y) d \sigma \leq \int_{0}^{s} v^{*}(\sigma, y) d \sigma, \quad \forall(s, y) \in I_{l} \times \Omega^{\prime \prime} .
$$

Proof: Let $u^{\epsilon}$ be the sequence given in Lemma 7 . The results proved in $[\mathbf{2}]$ and $[\mathbf{1 3}]$ ensure that

$$
\begin{array}{r}
-\left.\frac{\partial u^{\epsilon *}}{\partial s}\right|_{s=l}-g(l)\left(b_{h k}(y)\left(\int_{0}^{l} u^{\epsilon *}(\sigma, y) d \sigma\right)_{y_{h}}\right)_{y_{k}} \\
\leq \lambda_{1}^{\epsilon} g(l) \int_{0}^{l} u^{\epsilon *}(\sigma, y) d \sigma \text { in } \Omega^{\prime \prime}
\end{array}
$$

and since

$$
-\left.\frac{\partial u^{\epsilon *}}{\partial s}\right|_{s=l} \geq 0, \quad \forall \epsilon>0,
$$


we deduce

$$
\begin{cases}-\left(b_{h k}(y)\left(\int_{0}^{l} u^{\epsilon *}(\sigma, y) d \sigma\right)_{y_{h}}\right)_{y_{k}} \leq \lambda_{1}^{\epsilon} \int_{0}^{l} u^{\epsilon *}(\sigma, y) d \sigma & \text { in } \Omega^{\prime \prime} \\ \int_{0}^{l} u^{\epsilon *}(\sigma, y) d \sigma=0 & \text { on } \partial \Omega^{\prime \prime} .\end{cases}
$$

By Lemma 7 there exists a constant $M>0$ such that

$$
0 \leq \lambda_{1}^{\epsilon} \int_{0}^{l} u^{\epsilon *}(\sigma, y) d \sigma<M, \quad \forall \epsilon>0 \text { and } \forall y \in \overline{\Omega^{\prime \prime}} .
$$

This implies

$$
0 \leq \int_{0}^{l} u^{\epsilon *}(\sigma, y) d \sigma \leq \Phi(s, y), \quad \forall \epsilon>0 \text { and } \forall y \in \overline{\Omega^{\prime \prime}},
$$

where $\Phi$ is the solution of

$$
\begin{cases}-\left(b_{h k}(y) \Phi_{y_{h}}\right)_{y_{k}}=M & \text { in } \Omega^{\prime \prime} \\ \Phi=0 & \text { on } \partial \Omega^{\prime \prime}\end{cases}
$$

We have proved that the sequence $\int_{0}^{l} u^{\epsilon *}(\sigma, y) d \sigma$ is uniformly (with respect to $\epsilon$ and $y$ ) Lipschitz continuous up to $\partial \Omega^{\prime \prime}$. On the other hand, using again the Hopf maximum principle, we get

$$
\left|D_{y}\left(\int_{0}^{l} v^{*}(\sigma, y) d \sigma\right)\right| \geq C, \quad \forall y \in \partial \Omega^{\prime \prime},
$$

for some $C>0$.

We deduce that there exists a constant $C>0$, such that

$$
C_{\epsilon} \equiv \sup _{\overline{\Omega^{\prime \prime}}} \frac{\int_{0}^{l} u^{\epsilon *}(\sigma, y) d \sigma}{\int_{0}^{l} v^{*}(\sigma, y) d \sigma}>C, \quad \forall \epsilon>0 .
$$

This implies that the sequence

$$
\widetilde{u}^{\epsilon} \equiv C_{\epsilon}^{-1} u^{\epsilon}
$$

verifies

$$
\int_{0}^{l} \widetilde{u}^{\epsilon *}(\sigma, y) d \sigma \leq \int_{0}^{l} v^{*}(\sigma, y) d \sigma, \quad \forall \epsilon>0 \text { and } \forall y \in \Omega^{\prime \prime}
$$


Letting $\epsilon$ goes to $0^{+}$, from (4.28) and Lemma 7, we get (4.21). Finally, repeating the arguments of Theorem 3 , we get

$$
\int_{0}^{s} \widetilde{u}^{\epsilon *}(\sigma, y) d \sigma \leq \int_{0}^{s} v^{*}(\sigma, y) d \sigma, \quad \forall \epsilon>0 \text { and } \forall(s, y) \in I_{l} \times \Omega^{\prime \prime},
$$

and again passing to the limit as $\epsilon$ goes to $0^{+}$, we get the claim (4.22).

Remark 2. The result stated in Theorem 4, together with Remarks 3.2 and 3.3 of [2], allows to estimate the first eigenfunction of the Laplacian when $\Omega$ has cross sections of constant measure, for instance, in polar coordinates. Consider, for the sake of simplicity, domains in $\mathbb{R}^{2}$ as in Figure 1 below. In this case, we can apply Theorem 4 to the Laplace operator written in polar coordinates

$$
-\frac{1}{\rho} \frac{\partial}{\partial \rho}\left(\rho \frac{\partial u}{\partial \rho}\right)-\frac{1}{\rho^{2}} \frac{\partial^{2} u}{\partial \theta^{2}}=\lambda u .
$$

Using a Steiner symmetrization with respect to the angular variable $\theta$, the symmetrized domain, see Figure 2, will be in the form

$$
\Omega^{\star}=\left\{(r, \theta) \in \mathbb{R}^{2}: \theta_{1}<\theta<\theta_{2}, r_{1}<r<r_{2}\right\}
$$

and the relative first eigenfunction is

$$
v(r, \theta)=\sin \frac{\pi\left(\theta-\theta_{1}\right)}{\theta_{2}-\theta_{1}}\left(\frac{J_{0}\left(k_{0} r\right)}{J_{0}\left(k_{0} r_{1}\right)}-\frac{N_{0}\left(k_{0} r\right)}{N_{0}\left(k_{0} r_{1}\right)}\right),
$$

where $N_{0}$ is the Neumann function and $k_{0}$ is the root of

$$
J_{0}\left(k r_{1}\right) N_{0}\left(k r_{2}\right)-J_{0}\left(k r_{2}\right) N_{0}\left(k r_{1}\right)=0 .
$$

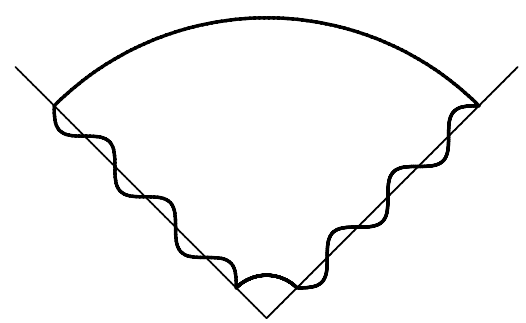

Figure 1

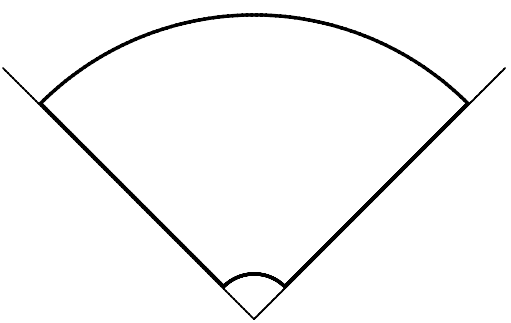

Figure 2

Acknowledgements. This work has been partially supported by GMAMPA - INDAM, Progetto "Proprietà analitico geometriche di soluzioni di equazioni ellittiche e paraboliche". 


\section{References}

[1] A. Alvino, V. Ferone, and G. Trombetti, On the properties of some nonlinear eigenvalues, SIAM J. Math. Anal. 29(2) (1998), 437-451 (electronic).

[2] A. Alvino, G. Trombetti, J. I. Díaz, and P. L. Lions, Elliptic equations and Steiner symmetrization, Comm. Pure Appl. Math. 49(3) (1996), 217-236.

[3] M. S. Ashbaugh and R. D. Benguria, A sharp bound for the ratio of the first two eigenvalues of Dirichlet Laplacians and extensions, Ann. of Math. (2) 135(3) (1992), 601-628.

[4] M. S. Ashbaugh and R. D. Benguria, A sharp bound for the ratio of the first two Dirichlet eigenvalues of a domain in a hemisphere of $S^{n}$, Trans. Amer. Math. Soc. 353(3) (2001), 1055-1087.

[5] A. BAernstein, II, A unified approach to symmetrization, in: "Partial differential equations of elliptic type" (Cortona, 1992), Sympos. Math. XXXV, Cambridge Univ. Press, Cambridge, 1994, pp. $47-91$.

[6] C. BANDLE, "Isoperimetric inequalities and applications", Monographs and Studies in Mathematics 7, Pitman (Advanced Publishing Program), Boston, Mass.-London, 1980.

[7] M. F. Betta, F. Chiacchio, And A. Ferone, Isoperimetric estimates for the first eigenfunction of a class of linear elliptic problems, Z. Angew. Math. Phys. 58(1) (2007), 37-52.

[8] B. Brandolini And C. Trombetti, A symmetrization result for Monge-Ampère type equations, Math. Nachr. 280(5-6) (2007), 467-478.

[9] A. Burchard, Steiner symmetrization is continuous in $W^{1, p}$, Geom. Funct. Anal. 7(5) (1997), 823-860.

[10] G. Chiti, A reverse Hölder inequality for the eigenfunctions of linear second order elliptic operators, Z. Angew. Math. Phys. 33(1) (1982), 143-148.

[11] G. ChIti, An isoperimetric inequality for the eigenfunctions of linear second order elliptic operators, Boll. Un. Mat. Ital. A (6) 1(1) (1982), 145-151.

[12] A. Cianchi and N. Fusco, Steiner symmetric extremals in PólyaSzegö type inequalities, Adv. Math. 203(2) (2006), 673-728.

[13] V. Ferone and A. Mercaldo, A second order derivation formula for functions defined by integrals, C. R. Acad. Sci. Paris Sér. I Math. 326(5) (1998), 549-554. 
[14] D. Gilbarg and N. S. Trudinger, "Elliptic partial differential equations of second order", Reprint of the 1998 edition, Classics in Mathematics, Springer-Verlag, Berlin, 2001.

[15] G. H. Hardy, J. E. Littlewood, and G. Pólya, "Inequalities", 2d ed., Cambridge, at the University Press, 1952.

[16] A. Henrot, "Extremum problems for eigenvalues of elliptic operators", Frontiers in Mathematics, Birkhäuser Verlag, Basel, 2006.

[17] B. KAwOHL, "Rearrangements and convexity of level sets in PDE", Lecture Notes in Mathematics 1150, Springer-Verlag, Berlin, 1985.

[18] S. Kesavan, "Symmetrization \& applications", Series in Analysis 3, World Scientific Publishing Co. Pte. Ltd., Hackensack, NJ, 2006.

[19] J. Mossino, "Inégalités isopérimétriques et applications en physique", Travaux en Cours. [Works in Progress], Hermann, Paris, 1984.

[20] B. Opic And A. Kufner, "Hardy-type inequalities", Pitman Research Notes in Mathematics Series 219, Longman Scientific \& Technical, Harlow, 1990.

[21] G. Pólya And G. Szegö, "Isoperimetric Inequalities in Mathematical Physics", Annals of Mathematics Studies 27, Princeton University Press, Princeton, N. J., 1951.

[22] G. Talenti, Elliptic equations and rearrangements, Ann. Scuola Norm. Sup. Pisa Cl. Sci. (4) 3(4) (1976), 697-718.

[23] G. Trombetтi, Symmetrization methods for partial differential equations, Boll. Unione Mat. Ital. Sez. B Artic. Ric. Mat. (8) 3(3) (2000), 601-634.

Dipartimento di Matematica e Applicazioni "R. Caccioppoli"

Università di Napoli "Federico II"

Complesso Monte S. Angelo - Via Cintia

80126 Napoli

Italy

E-mail address: francesco.chiacchio@unina.it

Rebut el 18 de maig de 2007. 